Article

\title{
Innovative Alcoholic Drinks Obtained by Co-Fermenting Grape Must and Fruit Juice
}

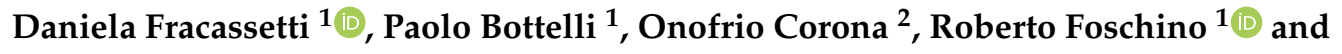 \\ Ileana Vigentini ${ }^{1, *}$ \\ 1 Department of Food, Environmental and Nutritional Sciences, Università degli Studi di Milano, \\ Via G. Celoria 2, 20133 Milan, Italy; daniela.fracassetti@unimi.it (D.F.); paolo.bottelli@gmail.com (P.B.); \\ roberto.foschino@unimi.it (R.F.) \\ 2 Department of Agricultural, Food and Forest Sciences, University of Palermo, Viale delle Scienze, \\ 90128 Palermo, Italy; onofrio.corona@unipa.it \\ * Correspondence: ileana.vigentini@unimi.it; Tel.: +39-0250319165; Fax: +39-0250319128
}

Received: 20 April 2019; Accepted: 27 April 2019; Published: 30 April 2019

\begin{abstract}
In this study, Cabernet Sauvignon and Chardonnay musts, and fruit juices from cherry, kiwi, peach, and strawberry were co-fermented with Saccharomyces cerevisiae EC1118 and Torulaspora delbrueckii UMY196 at two different proportions $(80: 20(v / v)$ and 60:40 $(v / v))$. The most pleasant fruit-based drink was obtained with Cabernet Sauvignon must and kiwi juice in a proportion of 60:40 and fermented with $T$. delbrueckii. This beverage was produced in higher volume to simulate a scale-up, and the aromatic profile, sensory description, and consumer acceptability were determined. The most powerful odorants of the kiwi-based drink were ethyl octanoate, phenylethanal, ethyl hexanoate, vinyl-guaiacol, benzaldehyde, and nonanal, for which the odor activity values were 21.1, $3.3,2.6,2.2,1.9$, and 1.6, respectively. These findings were in accordance with the sensory analysis, since the emerged descriptors were fruity (ethyl octanoate), honey and floral (phenylethanal), apple and peach (ethyl hexanoate), and citrus (nonanal). The consumers judged the kiwi-based drink acceptable (67\%) and 39\% of them would buy it. The reliable fermentation of a grape must/fruit juice was demonstrated. The kiwi-based drink represents an innovative and pleasant beverage with a positive impact on sustainability as its production can limit the loss of fresh fruits, as well as contribute to the enological field.
\end{abstract}

Keywords: fruit wines; food innovation; yeasts; secondary metabolites; grape must; kiwi juice

\section{Introduction}

Innovation in the sector of alcoholic beverages using sustainable approaches is a challenge from both environmental and productive points of view. Indeed, possible solutions consider several aspects including waste re-conversion or re-use, social impact (i.e., reduction of food loss, production of low-alcohol beverages), and economic advantage (i.e., decrease in wine consumption, unsold wine). Wine is an alcoholic beverage obtained by yeast fermentation of a purely grape must of Vitis vinifera vine species. The art of winemaking started back ca. 6000-5800 before Christ (BC) during the early Neolithic Period in Georgia in the South Caucasus region [1], while alternative alcoholic beverages from hawthorn fruit, rice, and honey mead were already produced as early as ca. $7000 \mathrm{BC}$ in ancient China [2]. The resulting alcoholic products from fruits other than grapes are called "fruit wines" and they show differences in taste, nutritive values, and health benefits [3].

In the last 15 years, global wine consumption went up and down [4]. Europe is consuming less and less wine, while the wine consumption in young producing areas is still growing. New alternative wines are appearing on the market and they are cheap and easy-drinking. Examples are the "flavored" 
wines (red lollipop, peach, grapefruit, mandarin, or black currant) with medium alcohol content (from $8 \%$ to $10.5 \%$ ) obtained by blending wines and fruit juices or flavoring wines with artificial or natural aromas. The main consumers of these products are younger people between 18 and 34 years old, with $33 \%$ drinking flavored wines as an aperitif.

Fruit wines are produced from fruit juices other than grape, such as apples, apricots, berries, cherries, plums, strawberries, oranges, mangoes, bananas, and pineapples [5,6], as well as autochthonous Brazilian fruits [7]. Moreover, the production implies the use of fruit juice concentrates, which allows increasing ethanol yield and taste, aroma, and functional features [8]. The production of alcoholic beverages obtained from the co-fermentation of grape must and fruit juice is yet to be investigated. Advantages of this approach are ascribable to an ever-growing rate of global food consumption, whereby the food supply production needs to fulfil all requests in a sustainable way in terms of environmental soundness, social equity, and economic feasibility. The formulation of new mixed-fruit alcoholic beverages could represent a reduction in fruit surplus and post-harvest fruit loss, and it could positively contribute to the economy of the existing wine industry. The reduction of food loss and wastes is gaining increasing importance [9] for increasing the food chain sustainability, even with the production of novel foods. The fermentation of fruit juices using selected yeasts can yield final products enriched in novel bio-functional compounds not found in traditional wines [3].

This study aimed to investigate, from microbial, chemical, and sensory points of view, the co-fermentation of grape musts and fruit juices obtained from cherries, kiwi, peaches, and strawberries. Alcoholic fermentations were carried out inoculating either Saccharomyces cerevisiae or the non-Saccharomyces species, Torulaspora delbrueckii, in four blends of grape musts (Chardonnay and Cabernet Sauvignon) and juices in different proportions. Consumer acceptability was also evaluated. The novel products could be produced using the surplus of some agriculture systems, such as spring/summer fruits and grape.

\section{Results and Discussion}

\subsection{Flask Trials}

\subsubsection{Fermentation Trends and Chemical Composition}

The trend of alcoholic fermentation (AF) was monitored for each must/fruit juice mix. T. delbrueckii showed a lower fermentative vigor in comparison to $S$. cerevisiae (Figure 1) as previously found in grape must fermentation [10]. S. cerevisiae started the AF in $24 \mathrm{~h}$, producing the following averages: (i) from Cabernet musts/fruit-based mixes, $10.90 \pm 0.40$ and $12.55 \pm 0.70 \mathrm{~g} \mathrm{CO}_{2} / \mathrm{L}$ in 80:20 and 60:40 proportions, respectively; (ii) from Chardonnay musts/fruit-based mixes, $12.35 \pm 0.95$ and $13.55 \pm 0.08 \mathrm{~g} \mathrm{CO}_{2} / \mathrm{L}$ in 80:20 and 60:40 proportions, respectively. The AF started in $48 \mathrm{~h}$ with $\mathrm{T}$. delbrueckii reaching similar values of $\mathrm{CO}_{2} / \mathrm{L}$ as the corresponding $S$. cerevisiae trials: (i) from Cabernet musts/fruit-based mixes, $10.49 \pm 3.35$ and $13.84 \pm 4.39 \mathrm{~g} \mathrm{CO}_{2} / \mathrm{L}$ in 80:20 and 60:40 proportions, respectively; (ii) from Chardonnay musts/fruit-based mixes, $11.55 \pm 2.73$ and 13.26 $\pm 2.16 \mathrm{~g} \mathrm{CO}_{2} / \mathrm{L}$ in 80:20 and 60:40 proportions, respectively. While the AF carried out with $S$. cerevisiae ended in 3-10 days, depending on the grape/fruit-based mix, T. delbrueckii completed the AF in a longer time (9-33 days) (Figure 1). S. cerevisiae showed a comparable fermentative profile for all types of fruit. In the case of T. delbrueckii, the fermentative trend with kiwi was significantly slower, regardless of the grape-kiwi juice proportion and combination. Since all inocula were standardized at the same cell concentration $\left(1 \times 10^{6}\right.$ colony-forming units (CFU)/mL) (data not shown), this result needs further investigation. 

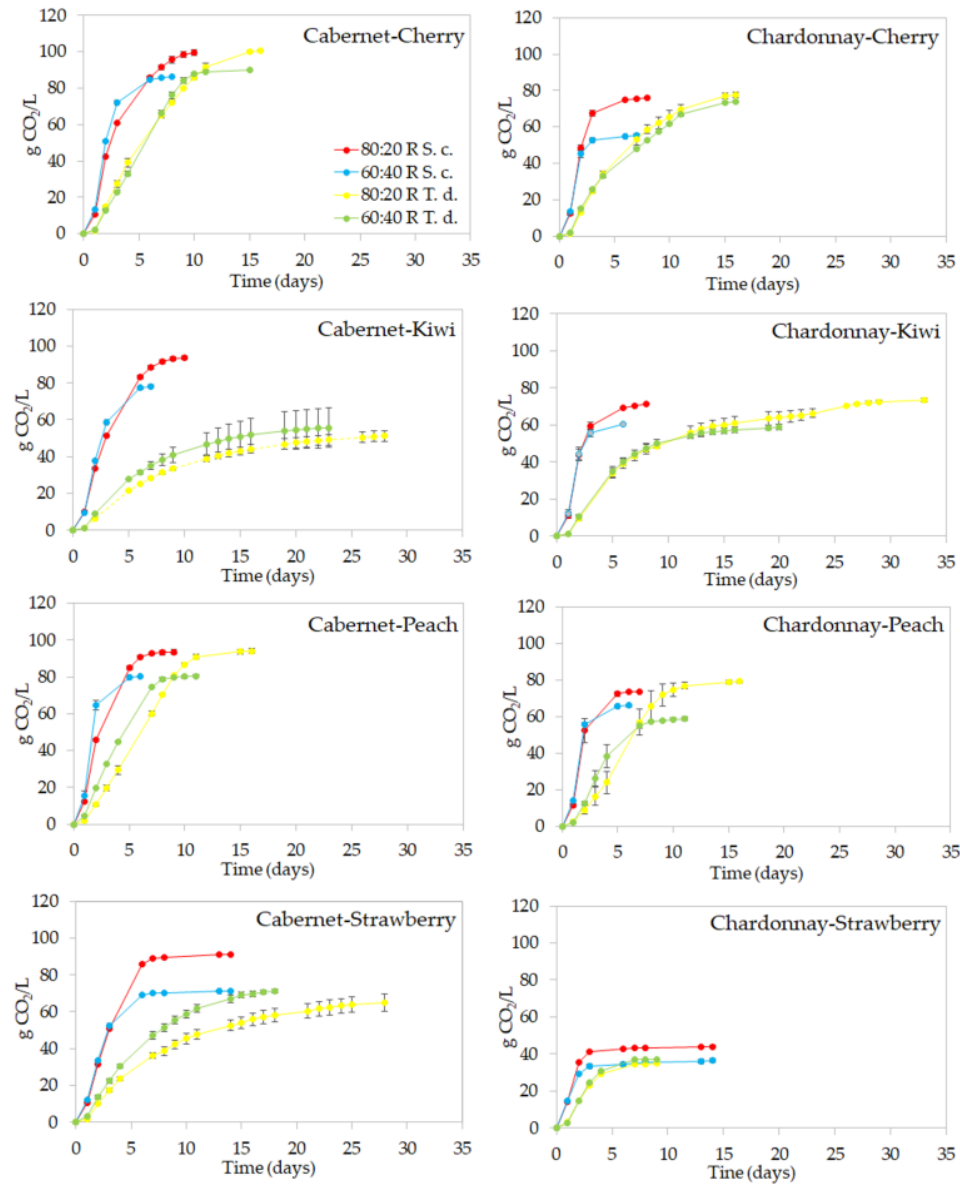

Figure 1. Alcoholic fermentation trends for the trials in flasks of different combinations must/fruit juice inoculated with Saccharomyces cerevisiae EC1118 (S. c.) and Torulaspora delbrueckii UMY196 (T. d.). Error bars indicate the standard deviation among replicates.

The residual sugars were about $3 \mathrm{~g} / \mathrm{L}$, except for the kiwi-based drinks obtained with Cabernet Sauvignon must and fermented with T. delbrueckii (Table 1). A higher content of residual sugars and a slower fermentation can have a positive impact on the aromatic profile [10]. The ethanol yield was comparable between T. delbrueckii and S. cerevisiae (Table S2, Supplementary Materials); this is of interest for the production of quality wines where the former species could be used as a starter culture [11].

Table 1. Chemical parameters determined for fermentation trial with grape must/kiwi juice fermented with Saccharomyces cerevisiae EC1118 (S. c.) and Torulaspora delbrueckii UMY196 (T. d.).

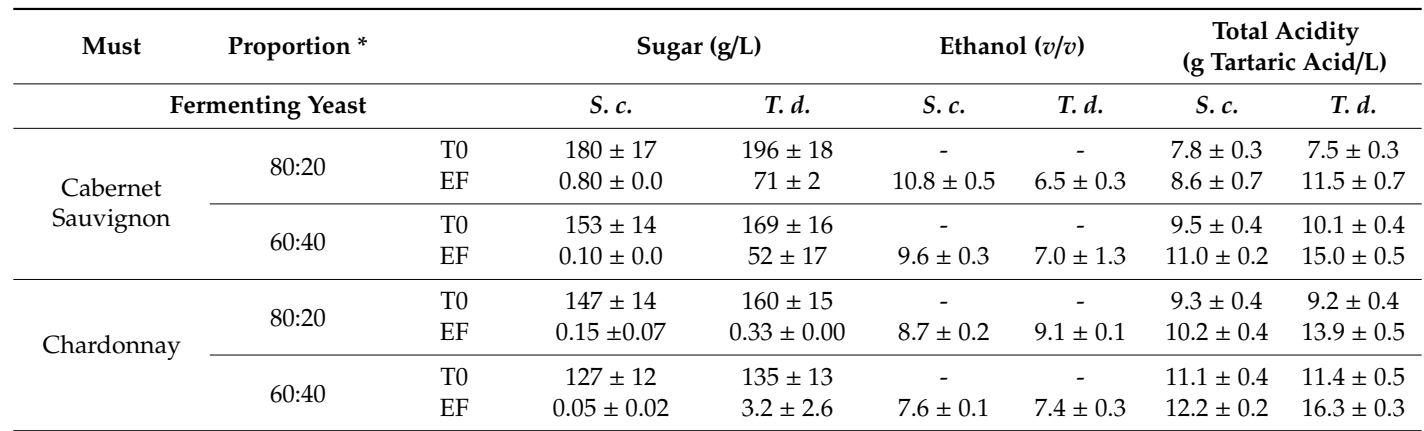

${ }^{*}$ The proportion is related to must/kiwi juice $(v / v)$. T0: concentrations of chemical parameters in kiwi juice/must; EF: concentrations of chemical parameters in grape/kiwi drink at the end of alcoholic fermentation. 
At the end of AF, the concentrations of sugar, ethanol, and organic acids, as well as the $\mathrm{pH}$ and total acidity, were determined; the characteristics of all final products are summarized in Table A1 and Figure A1 (Appendix A). The decrease in tartaric acid found for the cherry-, peach-, and kiwi-based drinks could be due to either salification or precipitation phenomena [12]. A drop of malic acid was detected particularly for the cherry-based drinks. This finding needs further investigation since the contribution of $S$. cerevisiae in malic acid decrease can be excluded because it lacks a specific system for malic acid transport. An increase in total acidity was found, especially in trials where T. delbrueckii was inoculated. This could be due to the higher concentrations of succinic acid responsible for an increase in titratable acidity during fermentation [13]. T. delbrueckii was a higher producer of succinic acid than S. cerevisiae during the fermentation of must [11]; further studies will be carried out to clarify the behavior of T. delbrueckii during the co-fermentation of grape must and fruit juice.

\subsubsection{Sensory Analysis}

The beverages obtained from co-fermenting Cabernet Sauvignon and cherry juice were particularly unpleasant with both yeasts (scores of overall acceptability <0.5/10) (Figure S1A, Supplementary Materials) due to the note of "chemical-medicinal" flavors. Drinks produced with Chardonnay and cherry juice received a better overall acceptability score, albeit still low (3/10) (Figure S1B, Supplementary Materials).

Most appreciated grape/fruit-based drinks resulted from with the fermentation of kiwi and Cabernet Sauvignon must, at the two proportions of 80:20 and 60:40, inoculated with a pure culture of T. delbrueckii. Indeed, these products received an average score of 5.4/10 and 4.7/10, respectively (Figure 2A). Their pleasantness was associated with a high perception of fruity aromas (4.5/10 and $3.8 / 10$, respectively) and sweetness (4.5/10 and 2.8/10, respectively). In agreement with McMahon and collaborators [14], the presence of sugars allows a decrease in bitterness and acidic taste perception. On the contrary, drinks from Chardonnay must and kiwi juice resulted unpleasant (Figure 2B).
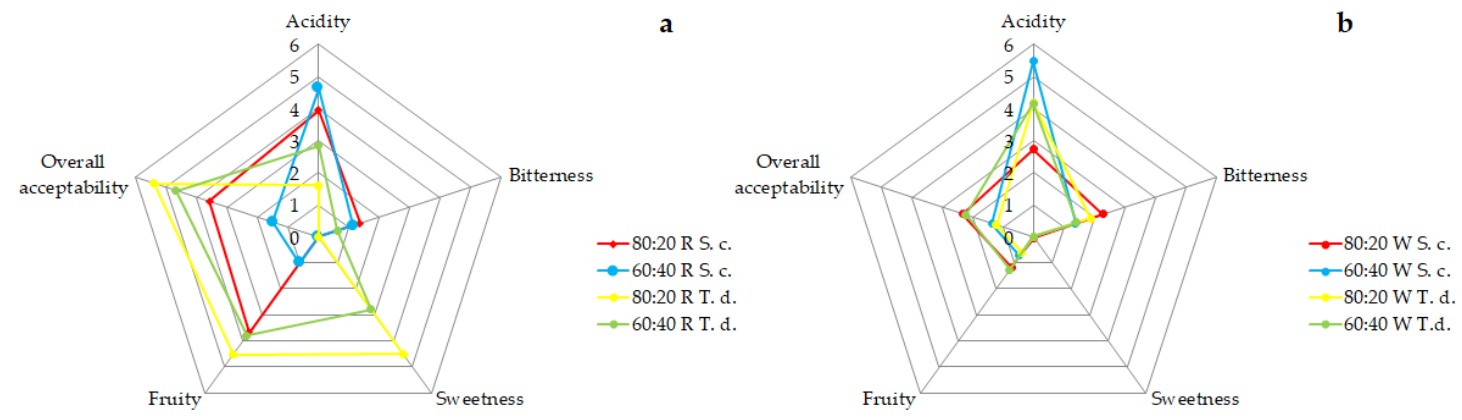

Figure 2. Gustatory profile of kiwi-based drinks for the trial in flasks fermented with (a) Saccharomyces cerevisiae EC1118 (S. c.), and (b) Torulaspora delbrueckii UMY196 (T. d.) with red (R) and white (W) grape musts. Data were obtained from medians of the scores indicated by the judges.

A generally low taste score (2.4/10) was assigned to drinks produced with peach juice (Figure S1C,D, Supplementary Materials). The least pleasant combinations were obtained with Chardonnay must at an 80:20 proportion (0.64/10) fermented with both yeasts, separately, for which the descriptor of "chemical-solvent" flavor was indicated.

For the strawberry-based drinks, the highest scores were related to the perception of acidity. Although the fruity note was also perceived, the overall acceptability was very low $(0.45 / 10)$ (Figure S1E,F, Supplementary Materials). In combination with Cabernet Sauvignon, it emerged that the bitterness negatively affected the overall acceptability.

Based on the sensory evaluation, the drink from the Cabernet Sauvignon must/kiwi juice mix at the proportion of 60:40 fermented by T. delbrueckii was selected as the most promising novel beverage and it was replicated in batches $(0.6 \mathrm{~L})$ and in microvinification $(4 \mathrm{~L})$. 


\subsection{Batch Experiment}

\subsubsection{Alcoholic Fermentation Trend}

The Cabernet Sauvignon/kiwi blend had the chemical characteristics shown in Table 2. The yeast inoculum resulted similar to the one performed during the preparation of flasks $\left(5.8 \pm 1.6 \times 10^{6} \mathrm{vs}\right.$. $6.2 \pm 1.6 \times 10^{6} \mathrm{CFU} / \mathrm{mL}$ ). A higher amount of $\mathrm{g} \mathrm{CO}_{2} / \mathrm{L}$ was developed in $48 \mathrm{~h}$ during the fermentation in batch in comparison to the fermentation in flask $\left(28.5 \pm 5.4 \mathrm{vs} .8 .97 \pm 0.53 \mathrm{~g} \mathrm{CO}_{2} / \mathrm{L}\right.$ produced in two days). The faster alcoholic fermentation could be due to the sampling, which was carried out by opening the bottles, leading to a possible aeration of must. This could favor the synthesis of essential fatty acids and sterols required for yeast replication [15] and, consequently, the fermentation rate could increase.

Table 2. Chemical parameters determined for batch fermentation with Cabernet Sauvignon must/kiwi juice 40:60 (v/v) fermented with Torulaspora delbrueckii UMY196.

\begin{tabular}{ccc}
\hline Chemical Parameter & Must/Kiwi Juice & Kiwi-Based Drink \\
\hline Sugar $(\mathrm{g} / \mathrm{L})$ & $190.1 \pm 8.0$ & $31.1 \pm 8.8$ \\
Ethanol $(v / v)$ & - & $7.6 \pm 0.4$ \\
pH & $3.2 \pm 0.0$ & $3.2 \pm 0.1$ \\
Total acidity (g tartaric acid/L) & $10.2 \pm 0.3$ & $14.9 \pm 1.4$ \\
Tartaric acid (g/L) & $1.70 \pm 0.06$ & $1.66 \pm 0.05$ \\
Malic acid (g/L) & $2.58 \pm 0.39$ & $3.00 \pm 0.22$ \\
Lactic acid (g/L) & n.d. & n.d. \\
Acetic acid (g/L) & n.d. & $0.21 \pm 0.06$ \\
Citric acid (g/L) & $6.03 \pm 0.22$ & $5.86 \pm 0.14$ \\
Succinic acid (g/L) & n.d. & $1.83 \pm 0.11$ \\
\hline
\end{tabular}

The trial was carried out in triplicate (volume: $0.6 \mathrm{~L}$ ); n.d.: not detected.

The fermentation was interrupted with a residual sugar content of $31.1 \pm 8.8 \mathrm{~g} / \mathrm{L}$, in agreement with the sugar amount found in the flask tests (Table 1), and with an ethanol value of $7.6 \pm 0.1 \%(\mathrm{v} / \mathrm{v})$ (Table 2). The tasting showed that batch and flask drinks were comparable (Figure 3).

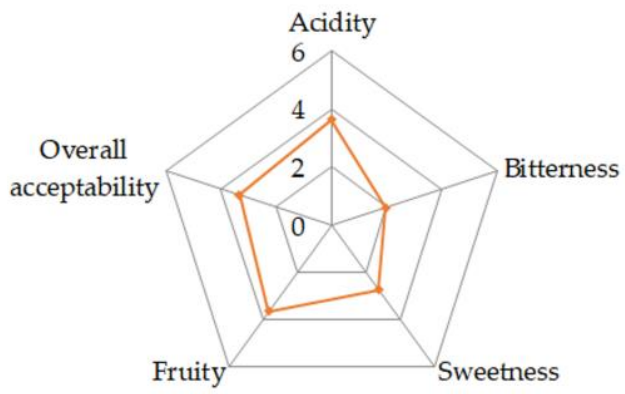

Figure 3. Gustatory profile of kiwi-based drink for the trial in batch fermented with Torulaspora delbrueckii UMY196.Data were obtained from medians of the scores indicated by the judges.

\subsubsection{Aroma Profile}

The contents of the aromatic compounds were monitored in the must/kiwi juice mix and during the $\mathrm{AF}$, on the third, seventh, and 17th days (decanting), allowing us to follow the evolution of aromas. Fifty-seven free aroma compounds were detected (Table 3). Except for aldehydes, the fermentation was fundamental for the aromatic complexity of the drink [16]. In fact, most of the free aromas were already found in the must/kiwi juice mix, and 33 free aromas (out of 57 compounds detected) increased just on the third day. Their concentrations were relatively unchanged during the fermentation with the exception of 3,4-dimethyl pentanol, phenylethyl acetate, ethyl hexadecanoate, ethyl hydrogen succinate, and cis-linalool oxide, for which higher amounts were found on the seventh day. For 
some of the free aromas, the slight decreases observed may be caused by the bottle opening for the sampling and, except for aldehydes and acids, they were not statistically significant. The most powerful odorants of the kiwi-based drink were ethyl octanoate, phenylethanal, ethyl hexanoate, vinyl-guaiacol, benzaldehyde, and nonanal, for which the odor activity values (OAVs) were 21.1, 3.3, 2.6, 2.2, 1.9, and 1.6, respectively. Ethyl octanoate and ethyl hexanoate are esters derived from the enzymatic activity of yeasts, confirming that the use of T. delbrueckii can improve the aromatic profile as occurs in wines $[10,16,17]$. Comparing the aromatic composition of wines obtained from Cabernet Sauvignon grapes and one of two kiwi fruit juices and purees $[18,19]$, we can hypothesize that phenylethanal and nonanal derive from kiwi juice, as they were not present in Cabernet Sauvignon wines analyzed by the cited authors. All the other compounds originated from the fermentative activity of yeasts, since varietal aromas specific to the Cabernet Sauvignon variety were not detected. This highlights that kiwi juice can positively influence the aromatic profile of the final grape/kiwi-based drink.

Table 3. Evolution of free aromatic compounds determined for the batch fermentation with Cabernet Sauvignon must/kiwi juice 60:40 (v/v) fermented with Torulaspora delbrueckii UMY196.

\begin{tabular}{|c|c|c|c|c|c|c|}
\hline \multirow{2}{*}{ Compound } & \multirow{2}{*}{$\begin{array}{c}\text { Perception } \\
\text { Threshold } \\
(\mu \mathrm{g} / \mathrm{L})\end{array}$} & \multirow{2}{*}{ Descriptor } & \multirow{2}{*}{$\begin{array}{l}\text { Cabernet Sauvignon } \\
\text { Must/Kiwi Juice }\end{array}$} & \multicolumn{3}{|c|}{ Days of Fermentation } \\
\hline & & & & 3 & 11 & $17 *$ \\
\hline \multicolumn{7}{|l|}{ Acids } \\
\hline Isobutyric acid & $2300^{c}$ & $\begin{array}{l}\text { Rancid, } \\
\text { butter, } \\
\text { cheese }\end{array}$ & $4.12 \pm 1.04$ & $89.77 \pm 78.06$ & n.d. & n.d. \\
\hline Isopentanoic acid & $33^{\mathrm{e}}$ & $\begin{array}{l}\text { Sweat, } \\
\text { rancid }\end{array}$ & n.d. & $100.72 \pm 95.63$ & $35.92 \pm 2.95$ & $36.41 \pm 6.74$ \\
\hline Pentanoic acid & $17^{\mathrm{d}}$ & Sweat & n.d. & $3.84 \pm 0.94$ & $3.00 \pm 1.25$ & $2.00 \pm 0.59$ \\
\hline Hexanoic acid & $420^{c}$ & Sweat & $32.02 \pm 11.50$ & $603.73 \pm 57.96$ & $465.00 \pm 51.79$ & $388.51 \pm 33.64$ \\
\hline $\begin{array}{l}\text { trans-2-Hexenoic } \\
\text { acid }\end{array}$ & - & Must, fat & $25.31 \pm 8.52$ & $42.69 \pm 10.35$ & $35.55 \pm 5.43$ & $27.84 \pm 2.18$ \\
\hline Octanoic acid & $500^{c}$ & $\begin{array}{l}\text { Cheese, } \\
\text { sweat }\end{array}$ & $13.13 \pm 0.87$ & $563.38 \pm 15.85$ & $479.39 \pm 43.96$ & $455.36 \pm 29.24$ \\
\hline Decanoic acid & $1000^{d}$ & Rancid, fat & n.d. & $319.58 \pm 32.81$ & $155.78 \pm 41.90$ & $112.86 \pm 54.34$ \\
\hline 9-Decenoic acid & $2^{d}$ & Fat & n.d. & $112.98 \pm 64.23$ & $79.20 \pm 14.41$ & $71.54 \pm 33.18$ \\
\hline $\begin{array}{l}\text { 2-Methylbutanoic } \\
\text { acid }\end{array}$ & $33^{d}$ & $\begin{array}{l}\text { Cheese, } \\
\text { sweat }\end{array}$ & n.d. & $130.16 \pm 21.04$ & $125.10 \pm 16.46$ & $89.07 \pm 21.52$ \\
\hline 2-Butenoic acid & - & Milky & $52.51 \pm 9.48$ & $72.45 \pm 8.05$ & $76.33 \pm 22.06$ & $60.12 \pm 8.34$ \\
\hline Total & & & $127.09 \pm 31.41$ & $2039.31 \pm 384.93$ & $1455.26 \pm 200.20$ & $1243.71 \pm 189.78$ \\
\hline \multicolumn{7}{|l|}{ Alcohols } \\
\hline Isobutanol & $40,000^{\mathrm{d}}$ & $\begin{array}{l}\text { Wine, } \\
\text { solvent, } \\
\text { bitter }\end{array}$ & $60.27 \pm 9.53$ & $138.66 \pm 17.84$ & $94.70 \pm 18.77$ & $76.05 \pm 7.76$ \\
\hline 3-Penten-2-ol & - & Green, vinyl & $7.70 \pm 1.42$ & $32.98 \pm 15.11$ & $32.91 \pm 7.54$ & $27.04 \pm 6.73$ \\
\hline 1-Pentanol & - & Balsamic & n.d. & $10.62 \pm 1.70$ & $6.72 \pm 1.80$ & $5.83 \pm 0.96$ \\
\hline 1-Hexanol & $1110^{g}$ & $\begin{array}{c}\text { Resin, } \\
\text { flower, green }\end{array}$ & $48.55 \pm 15.96$ & $396.08 \pm 70.77$ & $328.74 \pm 23.61$ & $351.68 \pm 56.33$ \\
\hline 2-Hexanol & - & $\begin{array}{c}\text { Resin, } \\
\text { flower, green }\end{array}$ & $20.77 \pm 5.06$ & $29.54 \pm 3.40$ & $26.19 \pm 4.83$ & $24.54 \pm 0.64$ \\
\hline 3-Ethoxy-1-propanol & - & Fruit & n.d. & $184.45 \pm 28.60$ & $169.08 \pm 4.77$ & $152.96 \pm 19.25$ \\
\hline cis-3-Hexen-1-ol & $400^{\mathrm{e}}$ & Grass & $29.70 \pm 10.07$ & $35.87 \pm 2.94$ & $25.83 \pm 0.50$ & $20.34 \pm 2.47$ \\
\hline 2-Ethyl-1-decanol & - & Fat & $25.65 \pm 4.03$ & $20.32 \pm 14.26$ & $2.21 \pm 0.48$ & $2.29 \pm 0.21$ \\
\hline 4-Hepten-1-ol & - & $\begin{array}{c}\text { Green, } \\
\text { grassy odor }\end{array}$ & n.d. & $4.66 \pm 4.47$ & $4.54 \pm 2.23$ & $5.25 \pm 0.92$ \\
\hline Isoamyl alcohol & $30,000^{d}$ & $\begin{array}{c}\text { Spirit, } \\
\text { alcoholic }\end{array}$ & $52.30 \pm 6.27$ & $16569 \pm 1348$ & $12909 \pm 860$ & $11224 \pm 953$ \\
\hline 2-Methyl-4-octanol & - & Cucumber & n.d. & $4.85 \pm 1.19$ & $2.72 \pm 0.89$ & $4.28 \pm 0.44$ \\
\hline 2,3-Butanediol & - & Fruit, onion & n.d. & $2.49 \pm 1.62$ & $11.07 \pm 2.17$ & $10.71 \pm 0.78$ \\
\hline Linalool & $15^{\mathrm{d}}$ & $\begin{array}{c}\text { Flower, } \\
\text { lavender }\end{array}$ & n.d. & $8.39 \pm 4.80$ & $2.59 \pm 0.55$ & $2.78 \pm 0.40$ \\
\hline $\begin{array}{l}\text { 3,4-Dimethyl } \\
\text { pentanol }\end{array}$ & - & - & $2.95 \pm 1.24$ & $2.23 \pm 0.25$ & $107.98 \pm 11.40$ & $92.08 \pm 15.65$ \\
\hline
\end{tabular}


Table 3. Cont

\begin{tabular}{|c|c|c|c|c|c|c|}
\hline \multirow{2}{*}{ Compound } & \multirow{2}{*}{$\begin{array}{l}\text { Perception } \\
\text { Threshold } \\
(\mu \mathrm{g} / \mathrm{L})\end{array}$} & \multirow{2}{*}{ Descriptor } & \multirow{2}{*}{$\begin{array}{c}\text { Cabernet Sauvignon } \\
\text { Must/Kiwi Juice }\end{array}$} & \multicolumn{3}{|c|}{ Days of Fermentation } \\
\hline & & & & 3 & 11 & 17 * \\
\hline$\alpha$-Terpineol & $250^{d}$ & $\begin{array}{c}\text { Oil, anise, } \\
\text { mint }\end{array}$ & $8.08 \pm 1.04$ & $10.54 \pm 2.10$ & $9.31 \pm 2.06$ & $9.93 \pm 1.50$ \\
\hline 2-Phenyl-2-hexanol & - & - & n.d. & $6.33 \pm 3.12$ & $3.78 \pm 0.83$ & $4.65 \pm 0.82$ \\
\hline Citronellol & $100^{d}$ & Rose & n.d. & $3.26 \pm 0.46$ & $3.37 \pm 0.84$ & $3.72 \pm 1.37$ \\
\hline Geraniol & $30^{d}$ & $\begin{array}{c}\text { Rose, } \\
\text { geranium }\end{array}$ & n.d. & $9.32 \pm 2.71$ & $8.61 \pm 0.97$ & $8.54 \pm 1.08$ \\
\hline 2-Phenylethanol & $10,000^{\mathrm{d}}$ & $\begin{array}{l}\text { Honey, spice, } \\
\text { rose, lilac }\end{array}$ & n.d. & $15978 \pm 504$ & $16286 \pm 1805$ & $14660 \pm 690$ \\
\hline$p$-Tyrosol & - & - & n.d. & $258.13 \pm 59.94$ & $271.51 \pm 20.35$ & $223.25 \pm 13.96$ \\
\hline Total & & & $255.97 \pm 54.62$ & $33704 \pm 2089$ & $30307 \pm 2770$ & $26256 \pm 2174$ \\
\hline \multicolumn{7}{|l|}{ Aldehydes } \\
\hline Nonanal & $8^{d}$ & $\begin{array}{l}\text { Fat, citrus, } \\
\text { green }\end{array}$ & $66.71 \pm 22.51$ & $12.21 \pm 2.44$ & $10.99 \pm 1.30$ & $12.45 \pm 3.18$ \\
\hline Benzaldehyde & $5^{f}$ & $\begin{array}{l}\text { Almond, } \\
\text { sugar }\end{array}$ & $16.27 \pm 3.80$ & $7.78 \pm 3.27$ & $7.10 \pm 1.14$ & $9.26 \pm 4.13$ \\
\hline Phenylethanal & $1^{\mathrm{e}}$ & $\begin{array}{c}\text { Honey, } \\
\text { sweet, } \\
\text { hawthorn }\end{array}$ & n.d. & $5.73 \pm 2.88$ & $4.27 \pm 0.40$ & $3.25 \pm 1.24$ \\
\hline $\begin{array}{l}\text { 2,4-Dimethyl } \\
\text { benzaldehyde }\end{array}$ & - & Sweet & $4.37 \pm 1.11$ & $4.25 \pm 0.53$ & $6.41 \pm 2.61$ & $7.23 \pm 2.46$ \\
\hline Total & & & $87.35 \pm 27.42$ & $29.97 \pm 9.12$ & $28.77 \pm 5.45$ & $32.19 \pm 11.00$ \\
\hline \multicolumn{7}{|l|}{ Benzenoids } \\
\hline 4-Vinyl guaiacol & $40^{\mathrm{d}}$ & Clove, curry & $8.84 \pm 1.91$ & $100.32 \pm 3.32$ & $108.49 \pm 12.39$ & $83.19 \pm 8.35$ \\
\hline Guaiacol & $9.5^{\mathrm{e}}$ & $\begin{array}{l}\text { Smoke, } \\
\text { sweet, } \\
\text { medicine }\end{array}$ & $4.20 \pm 2.21$ & $10.45 \pm 2.97$ & $9.22 \pm 1.42$ & $6.10 \pm 3.94$ \\
\hline Syringol & - & $\begin{array}{l}\text { Medicine, } \\
\text { phenol, } \\
\text { smoke }\end{array}$ & $88.84 \pm 24.29$ & $65.25 \pm 2.79$ & $61.30 \pm 12.28$ & $51.05 \pm 7.30$ \\
\hline Total & & & $101.88 \pm 28.41$ & $176.01 \pm 9.08$ & $179.01 \pm 26.09$ & $146.28 \pm 19.59$ \\
\hline \multicolumn{7}{|l|}{ Esters } \\
\hline Isoamyl acetate & $12,270^{\mathrm{d}}$ & Banana & n.d. & $19.52 \pm 2.66$ & $17.18 \pm 1.29$ & $21.81 \pm 3.48$ \\
\hline Ethyl hexanoate & $14^{\mathrm{a}}$ & $\begin{array}{l}\text { Apple, } \\
\text { peach }\end{array}$ & n.d. & $59.77 \pm 3.26$ & $37.93 \pm 6.20$ & $35.84 \pm 3.75$ \\
\hline Ethyl octanoate & $2^{f}$ & Fruit, fat & n.d. & $47.79 \pm 7.05$ & $44.75 \pm 1.55$ & $42.12 \pm 2.66$ \\
\hline Ethyl decanoate & $200^{d}$ & Grape & $5.15 \pm 1.04$ & $105.10 \pm 57.31$ & $36.54 \pm 10.77$ & $29.11 \pm 16.62$ \\
\hline Diethyl succinate & $200,000^{\mathrm{e}}$ & Wine, fruit & n.d. & $6.21 \pm 2.14$ & $18.72 \pm 2.34$ & $26.21 \pm 2.96$ \\
\hline Ethyl-9-decenoate & - & Fruity & n.d. & $45.76 \pm 27.65$ & $26.45 \pm 14.37$ & $29.94 \pm 12.11$ \\
\hline Ethyl acetate & $7500^{d}$ & Pineapple & n.d. & $28.19 \pm 11.67$ & $15.78 \pm 4.43$ & $14.21 \pm 2.03$ \\
\hline$\alpha$-Isoamyl- $\gamma$-butyrolactone & - & $\begin{array}{l}\text { Coumarin, } \\
\text { sweet }\end{array}$ & n.d. & $33.19 \pm 6.15$ & $35.56 \pm 6.72$ & $30.75 \pm 3.19$ \\
\hline Phenylethyl acetate & $250^{d}$ & $\begin{array}{l}\text { Rose, honey, } \\
\text { tobacco }\end{array}$ & n.d. & $77.32 \pm 10.94$ & $109.03 \pm 18.45$ & $118.19 \pm 18.44$ \\
\hline Butyl isobutyrate & & $\begin{array}{c}\text { Fruity, green, } \\
\text { apple, } \\
\text { banana }\end{array}$ & n.d. & $112.44 \pm 13.31$ & $100.93 \pm 15.08$ & $82.38 \pm 9.51$ \\
\hline$\gamma$-Nonalactone & $25^{\mathrm{d}}$ & $\begin{array}{l}\text { Coconut, } \\
\text { peach }\end{array}$ & $4.71 \pm 2.87$ & $5.29 \pm 0.87$ & $6.24 \pm 0.99$ & $6.92 \pm 0.26$ \\
\hline Diethyl malate & - & $\begin{array}{l}\text { Brown sugar, } \\
\text { sweet }\end{array}$ & n.d. & $7.05 \pm 2.25$ & $7.47 \pm 0.63$ & $8.57 \pm 1.56$ \\
\hline $\begin{array}{c}\text { Methyl } \\
\text { hexadecanoate }\end{array}$ & - & Fat, wax & $44.59 \pm 6.38$ & $40.93 \pm 2.45$ & $37.31 \pm 4.58$ & $33.74 \pm 5.44$ \\
\hline $\begin{array}{c}\text { Ethyl } \\
\text { hexadecanoate }\end{array}$ & - & Wax & n.d. & $24.95 \pm 10.29$ & $70.72 \pm 16.86$ & $53.35 \pm 19.71$ \\
\hline $\begin{array}{l}\text { Ethyl hydrogen } \\
\text { succinate }\end{array}$ & - & Wine, fruit & n.d. & $43.36 \pm 29.15$ & $99.77 \pm 15.86$ & $87.70 \pm 19.43$ \\
\hline $\begin{array}{l}\text { Phenethyl } \\
\text { propionate }\end{array}$ & - & Fruit & n.d. & $18.16 \pm 2.35$ & $15.54 \pm 2.32$ & $14.09 \pm 1.21$ \\
\hline
\end{tabular}


Table 3. Cont

\begin{tabular}{|c|c|c|c|c|c|c|}
\hline \multirow{2}{*}{ Compound } & \multirow{2}{*}{$\begin{array}{c}\text { Perception } \\
\text { Threshold } \\
(\mu \mathrm{g} / \mathrm{L})\end{array}$} & \multirow{2}{*}{ Descriptor } & \multirow{2}{*}{$\begin{array}{l}\text { Cabernet Sauvignon } \\
\text { Must/Kiwi Juice }\end{array}$} & \multicolumn{3}{|c|}{ Days of Fermentation } \\
\hline & & & & 3 & 11 & 17 * \\
\hline Total & & & $54.46 \pm 10.28$ & $675.03 \pm 189.51$ & $679.92 \pm 122.45$ & $634.95 \pm 122.37$ \\
\hline \multicolumn{7}{|l|}{ Furanoids } \\
\hline cis-Linalool oxide & - & Flower & n.d. & $1.96 \pm 0.50$ & $197.44 \pm 27.48$ & $175.09 \pm 4.73$ \\
\hline \multicolumn{7}{|l|}{ Ketones } \\
\hline 6-Methyl-2-heptanone & - & Soap & n.d. & $8.16 \pm 4.18$ & $6.73 \pm 4.97$ & $6.01 \pm 1.16$ \\
\hline \multicolumn{7}{|l|}{ Norisoprenoids } \\
\hline 3-Hydroxy- $\beta$-damascone & - & $\begin{array}{c}\text { Apple, tea, } \\
\text { tobacco }\end{array}$ & $11.31 \pm 6.25$ & $146.28 \pm 106.57$ & $65.23 \pm 30.63$ & $87.69 \pm 8.09$ \\
\hline \multicolumn{7}{|l|}{ Thiols } \\
\hline 3-(Methylthio)-propanol & $1000^{b}$ & $\begin{array}{l}\text { Sweet, } \\
\text { potato }\end{array}$ & n.d. & $51.62 \pm 8.23$ & $63.92 \pm 6.73$ & $53.89 \pm 6.25$ \\
\hline
\end{tabular}

The trial was carried out in triplicate. ${ }^{*}$ Sampling at the end of fermentation; n.d.: not detected. References: ${ }^{a}$ [20];

${ }^{b}$ [21]; ${ }^{\mathrm{c}}[22]{ }^{\mathrm{d}}{ }^{\text {[23] }}{ }^{\mathrm{e}}$ [24]; ${ }^{\mathrm{f}}[25] ;{ }^{\mathrm{g}}$ [26].

Twenty glycosylated aroma compounds were detected and are listed in Table 4. Their concentrations decreased from the beginning of AF, in particular those of benzyl alcohol, 2-hexanal, 4 -vinyl guaicol, and 3-oxo- $\alpha$-ionol. Our findings suggest that the strain of T. delbrueckii used for the AF could perform a glycosidase activity, as reported in the literature [27]. Further investigation will be carried out to confirm this activity.

Table 4. Evolution of glycosylated aromatic compounds determined for the batch fermentation with Cabernet Sauvignon must/kiwi juice 60:40 (v/v) fermented with Torulaspora delbrueckii UMY196.

\begin{tabular}{|c|c|c|c|c|c|c|}
\hline \multirow{2}{*}{ Compound } & \multirow{2}{*}{$\begin{array}{c}\text { Perception } \\
\text { Threshold } \\
(\mu \mathrm{g} / \mathrm{L})\end{array}$} & \multirow{2}{*}{ Descriptor } & \multirow{2}{*}{$\begin{array}{l}\text { Cabernet Sauvignon } \\
\text { Must/Kiwi Juice }\end{array}$} & \multicolumn{3}{|c|}{ Days of Fermentation } \\
\hline & & & & 3 & 11 & $17^{*}$ \\
\hline \multicolumn{7}{|l|}{ Acids } \\
\hline Nonanoic acid & - & Green, fat & $20.20 \pm 1.22$ & $11.13 \pm 1.23$ & $17.10 \pm 2.61$ & $17.89 \pm 3.22$ \\
\hline Geranic acid & - & Green, floral & $28.77 \pm 9.87$ & $26.22 \pm 10.76$ & $21.65 \pm 10.08$ & $20.65 \pm 2.81$ \\
\hline Total & & & $48.97 \pm 11.09$ & $37.25 \pm 11.99$ & $38.75 \pm 12.69$ & $38.54 \pm 6.03$ \\
\hline \multicolumn{7}{|l|}{ Alcohols } \\
\hline 3-Penten-2-ol & - & Green, vinyl & $41.32 \pm 2.79$ & $41.41 \pm 4.60$ & $30.93 \pm 9.42$ & $38.67 \pm 6.20$ \\
\hline 1-Hexanol & $1110^{c}$ & $\begin{array}{c}\text { Resin, } \\
\text { flower, green }\end{array}$ & $34.95 \pm 4.95$ & $33.81 \pm 1.92$ & $32.93 \pm 5.49$ & $28.73 \pm 1.36$ \\
\hline 2-Hexanol & - & $\begin{array}{c}\text { Resin, } \\
\text { flower, green }\end{array}$ & $27.31 \pm 2.96$ & $24.95 \pm 0.83$ & $25.61 \pm 5.46$ & $26.56 \pm 3.20$ \\
\hline 3-Octanol & - & $\begin{array}{l}\text { Moss, nut, } \\
\text { mushroom }\end{array}$ & $79.09 \pm 4.60$ & $98.55 \pm 4.14$ & $99.64 \pm 2.55$ & $106.25 \pm 2.74$ \\
\hline Linalool & $15^{\mathrm{a}}$ & $\begin{array}{c}\text { Flower, } \\
\text { lavender }\end{array}$ & $12.10 \pm 2.69$ & $11.09 \pm 1.49$ & $10.19 \pm 0.78$ & $7.87 \pm 3.44$ \\
\hline$\alpha$-Terpineol & $250^{\mathrm{a}}$ & $\begin{array}{l}\text { Oil, anise, } \\
\text { mint }\end{array}$ & $9.34 \pm 1.07$ & $7.99 \pm 0.71$ & $9.21 \pm 0.40$ & $10.16 \pm 1.85$ \\
\hline Nerol & - & Sweet & $112.53 \pm 5.18$ & $125.19 \pm 8.13$ & $130.25 \pm 4.49$ & $131.72 \pm 13.58$ \\
\hline Benzyl alcohol & - & $\begin{array}{l}\text { Sweet, } \\
\text { flower }\end{array}$ & $334.71 \pm 13.38$ & $113.71 \pm 6.47$ & $110.66 \pm 8.20$ & $78.00 \pm 9.63$ \\
\hline 8-Hydroxygeraniol & - & - & $8.34 \pm 1.82$ & $8.38 \pm 1.22$ & $8.55 \pm 0.63$ & $16.24 \pm 3.92$ \\
\hline Total & & & $659.69 \pm 39.46$ & $465.08 \pm 29.51$ & $457.97 \pm 37.41$ & $444.20 \pm 45.92$ \\
\hline \multicolumn{7}{|l|}{ Aldehydes } \\
\hline 2-Hexanal & - & $\begin{array}{c}\text { Grass, tallow, } \\
\text { fat } \\
\end{array}$ & $164.99 \pm 19.71$ & $42.11 \pm 7.92$ & $34.88 \pm 8.33$ & $33.52 \pm 5.48$ \\
\hline Nonanal & $8^{a}$ & $\begin{array}{l}\text { Fat, citrus, } \\
\text { green }\end{array}$ & $4.31 \pm 1.16$ & $3.08 \pm 0.19$ & $2.45 \pm 0.57$ & $12.78 \pm 2.33$ \\
\hline Benzaldehyde & $5^{b}$ & $\begin{array}{l}\text { Almond, } \\
\text { sugar }\end{array}$ & $9.22 \pm 2.66$ & $4.09 \pm 2.22$ & $2.63 \pm 0.61$ & $2.52 \pm 0.91$ \\
\hline
\end{tabular}


Table 4. Cont.

\begin{tabular}{|c|c|c|c|c|c|c|}
\hline \multirow{2}{*}{ Compound } & \multirow{2}{*}{$\begin{array}{c}\text { Perception } \\
\text { Threshold } \\
(\mu \mathrm{g} / \mathrm{L})\end{array}$} & \multirow{2}{*}{ Descriptor } & \multirow{2}{*}{$\begin{array}{l}\text { Cabernet Sauvignon } \\
\text { Must/Kiwi Juice }\end{array}$} & \multicolumn{3}{|c|}{ Days of Fermentation } \\
\hline & & & & 3 & 11 & $17 *$ \\
\hline Total & & & $178.51 \pm 23.54$ & $49.28 \pm 10.34$ & $39.96 \pm 9.52$ & $48.82 \pm 8.72$ \\
\hline \multicolumn{7}{|l|}{ Benzenoids } \\
\hline 4-Vinyl guaiacol & $40^{\mathrm{a}}$ & Clove, curry & $154.10 \pm 36.96$ & $84.51 \pm 13.28$ & $100.87 \pm 10.01$ & $95.61 \pm 5.82$ \\
\hline Eugenol & - & Clove, honey & $14.20 \pm 2.32$ & $17.10 \pm 2.98$ & $16.96 \pm 1.85$ & $17.52 \pm 0.27$ \\
\hline Syringol & - & $\begin{array}{l}\text { Medicine, } \\
\text { phenol, } \\
\text { smoke }\end{array}$ & $49.85 \pm 19.09$ & $31.19 \pm 8.11$ & $58.55 \pm 22.44$ & $44.61 \pm 9.42$ \\
\hline Total & & & $218.16 \pm 58.37$ & $132.80 \pm 24.36$ & $176.38 \pm 34.29$ & $157.73 \pm 15.52$ \\
\hline \multicolumn{7}{|l|}{ Norisoprenoids } \\
\hline 3-oxo- $\alpha$-damascone & - & Apple & $35.24 \pm 11.16$ & $39.86 \pm 1.83$ & $38.49 \pm 3.05$ & $37.47 \pm 3.72$ \\
\hline 3-Oxo- $\alpha$-ionol & - & $\begin{array}{c}\text { Spice, tea, } \\
\text { tobacco }\end{array}$ & $118.34 \pm 32.57$ & $83.13 \pm 27.57$ & $81.43 \pm 2.23$ & $70.78 \pm 8.83$ \\
\hline Total & & & $153.58 \pm 58$ & $123.00 \pm 29.39$ & $119.92 \pm 5.28$ & $108.25 \pm 12.55$ \\
\hline \multicolumn{7}{|l|}{ Furanoids } \\
\hline cis-Linalool oxide & - & Flower & $11.18 \pm 1.72$ & $11.34 \pm 1.09$ & $12.41 \pm 0.24$ & $12.26 \pm 0.82$ \\
\hline
\end{tabular}

The trial was carried out in triplicate. ${ }^{*}$ Sampling at the end of fermentation; n.d.: not detected. References: ${ }^{\text {[ }}$ [23];

b $[25] ;{ }^{c}[26]$.

\subsection{Microvinification}

\subsubsection{Fermentation Trend and Chemical Composition}

The Cabernet Sauvignon/kiwi blend used for the microvinification experiments was characterized by slightly lower contents of sugar and tartaric acid (Table 5) in comparison to the mixture used for the batches and flasks trials; on the other hand, $\mathrm{pH}$ and total acidity resulted comparable. Possibly due to the fermentation temperature set at $18 \pm 1^{\circ} \mathrm{C}$, the trend of fermentation showed a more prolonged lag phase; indeed, only after five days of fermentation, a similar amount of $\mathrm{CO}_{2} / \mathrm{L}$ to that reached after $48 \mathrm{~h}$ in batch experiments was released $\left(27.5 \mathrm{~g} \mathrm{CO}_{2} / \mathrm{L}\right)$. However, once the AF started, the trend was comparable with fermentations in flask and in batches (data not shown).

Table 5. Chemical parameters determined for the microvinification trial with Cabernet Sauvignon must/kiwi juice 60:40 (v/v) fermented with Torulaspora delbrueckii UMY196.

\begin{tabular}{ccc}
\hline Chemical Parameter & Must/Kiwi Juice & Kiwi-Based Drink \\
\hline Sugar $(\mathrm{g} / \mathrm{L})$ & $180 \pm 17$ & $8.6 \pm 0.7$ \\
Ethanol $(\mathrm{v} / \mathrm{v})$ & - & $9.5 \pm 0.2$ \\
Methanol $(\mathrm{mg} / \mathrm{L})$ & - & 75.0 \\
pH & $3.3 \pm 0.01$ & $3.5 \pm 0.01$ \\
Total acidity $(\mathrm{g}$ tartaric acid/L) & $9.2 \pm 0.4$ & $9.4 \pm 0.6$ \\
Tartaric acid $(\mathrm{g} / \mathrm{L})$ & $0.79 \pm 0.03$ & $0.51 \pm 0.10$ \\
Malic acid $(\mathrm{g} / \mathrm{L})$ & $4.09 \pm 0.29$ & $2.63 \pm 0.54$ \\
Lactic acid $(\mathrm{g} / \mathrm{L})$ & n.d. & n.d. \\
Acetic acid $(\mathrm{g} / \mathrm{L})$ & n.d. & $0.11 \pm 0.07$ \\
Citric acid $(\mathrm{g} / \mathrm{L})$ & $5.37 \pm 0.28$ & $6.05 \pm 0.81$ \\
Succinic acid $(\mathrm{g} / \mathrm{L})$ & n.d. & n.d. \\
\hline
\end{tabular}

The trial was carried out in triplicate; n.d.: not detected.

\subsubsection{Chemical Composition of Kiwi-Based Drink}

The final product showed a residual sugar content equal to $8.6 \pm 0.7 \mathrm{~g} / \mathrm{L}$, lower than the predetermined target of $30 \mathrm{~g} / \mathrm{L}$ (Table 5 vs Table 2) and, consequently, with a higher ethanol concentration $(9.5 \pm 0.2 \%(v / v))$ in comparison to the flask and batch tests. Contrarily to what was found in the previous trials, a significant increase in total acidity was not detected; it was maintained at a value comparable to the grape/kiwi juice mixes (Table 5). Furthermore, contrarily to flask and batch experiments, succinic 
acid failed to be produced. The concentration of methanol was $75 \mathrm{mg} / \mathrm{L}$, lower than the law limit for wine fixed as $200 \mathrm{mg} / \mathrm{L}$ by the European Community [28].

\subsubsection{Sensory Analysis}

The descriptive-quantitative profile was evaluated for the final product from microvinification. Moreover, in order to reach the target amount of sugars $(30 \mathrm{~g} / \mathrm{L})$, reducing the perception of acidity, the drink produced with microvinification was supplemented with $22.4 \mathrm{~g} / \mathrm{L}$ sucrose. Since the aromas were already produced after three days of fermentation, it is plausible that the addition of sugar at this step could attenuate the perception of acidity and affect the aromatic profile to a relatively low extent. Nevertheless, further steps of scaling-up will optimize the technological operation to stop the fermentation at the required sugar content. The two kiwi-based drinks were tasted by an expert panel that identified the following descriptors: coppery color for the visual perception, peach, floral, passion fruit, and honey for the olfactory perception, and citrus, fruity, apple, and fruit salad for the retro-olfactory perception. Sweetness, acidity, bitterness, olfactory flavor intensity, viscosity, and aromatic intensity were also evaluated.

The selected descriptors resulted in accordance with the volatile compounds detected. Indeed, the fruity, honey and floral, apple and peach, and citrus notes, associated with ethyl octanoate, phenylethanal, ethyl hexanoate, and nonanal compounds, respectively, showed the higher OAVs (Section 2.2.2). Figure 4 highlights that the kiwi-based drinks had high olfactory and flavor intensities, indicating their aromatic richness and complexity. The kiwi-based drink added with sugar showed higher perceptions of sweetness, olfactory intensity, citrus and fruity notes, and minor acidity. However, these differences were not statistically significant, indicating the aromatic characteristics of the kiwi-based drink were not affected by its sweetness, as well as its complexity and richness, which were independent of the sugar content.

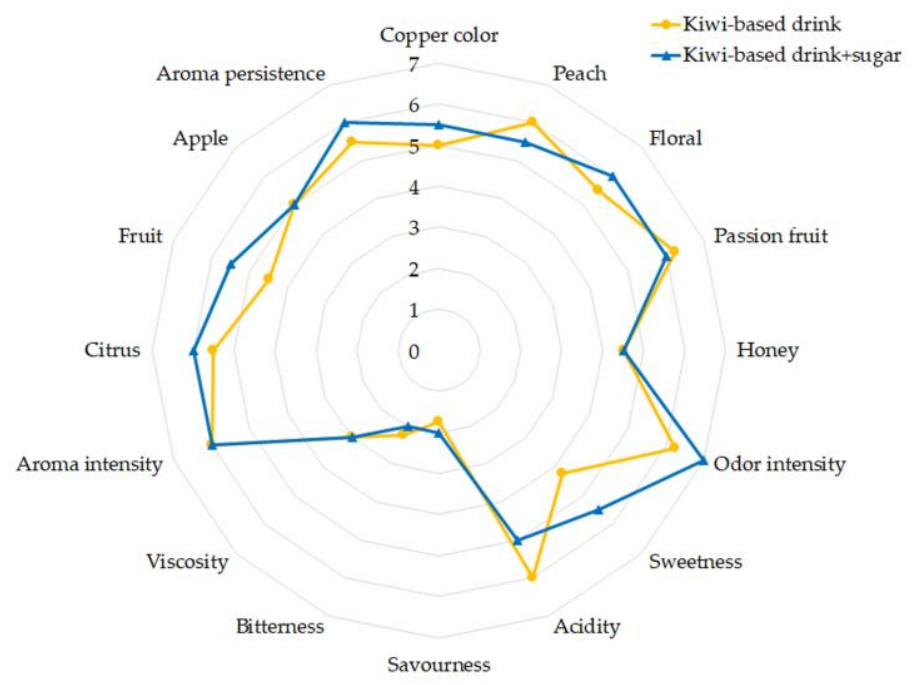

Figure 4. Descriptive-quantitative profile of kiwi-based drink for the trial in a glass demijohn fermented with Torulaspora delbrueckii UMY196.

\subsubsection{Consumer Acceptability}

The acceptability test was performed considering 100 consumers, asking them to judge both pleasantness and acceptability. The panel mainly constituted judges who are used to buying alcoholic beverages (72\%) and drinking them at least once a week (65\%). As Figure 5 showed, the Cabernet Sauvignon/kiwi drink was appreciated from the olfactory point of view (score 4/5). However, its flavor resulted unpleasant (score 2/5) due to the acidity and the lack of sweetness. Nevertheless, the score 
of global pleasantness was 3/5 as an average. In general, 67\% of consumers considered the product acceptable and $39 \%$ stated they would buy it.

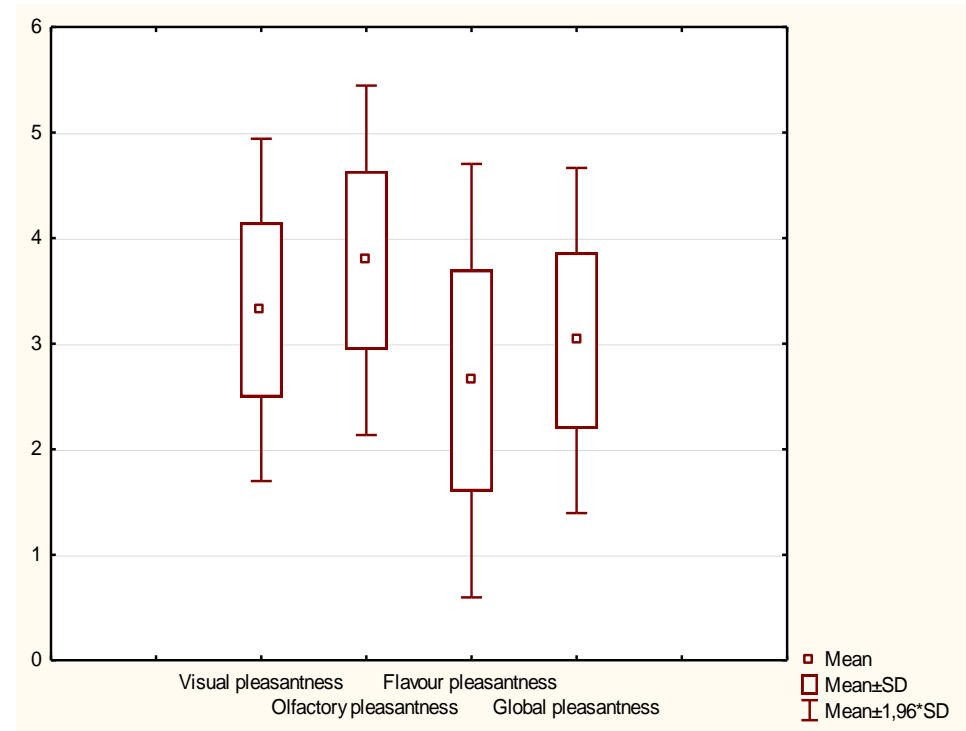

Figure 5. Box and wisker plot of the consumer acceptability test obtained for the kiwi-based drink for the trial in a glass demijohn fermented with Torulaspora delbrueckii UMY196.

\section{Materials and Methods}

\subsection{Preparation of Fruit Juices and Musts}

The two musts used in this study were industrially produced from Chardonnay and Cabernet Sauvignon grapes in two different wineries (Lombardia region, north of Italy). Chardonnay must was employed for the preliminary experiment; it was obtained in vintage 2015 using a Velvet 80 pneumatic press (DIEMME Enologia, Lugo, RA, Italy) working under nitrogen flow and without $\mathrm{SO}_{2}$, left for $12 \mathrm{~h}$ at $4{ }^{\circ} \mathrm{C}$ for settling and then stored at $-18 \pm 1^{\circ} \mathrm{C}$. The Cabernet Sauvignon must was produced in vintage 2015 for the preliminary and batch experiments and in vintage 2017 for the microvinification. The grape was crushed without sulfur dioxide $\left(\mathrm{SO}_{2}\right)$ and the grape skins were left in the must for three days. The liquid musts were collected and stored at $-18 \pm 1^{\circ} \mathrm{C}$.

The fruit juices were obtained from cherry (Prunus avium var. Durone nero di Vignola), kiwi (Actinidia chinenesis var. Gold), peach (Prunus persica var. Nucipersica), and strawberry (Fragaria ananassa var. Nabila). In the case of kiwi, a further amount of juice was produced for microvinification experiments. The ripe fruits of Italian origin were collected at a local farmer market and they were washed, gently dried, and cut (prior to seed removal) or peeled after purchasing. The fruit juices were obtained with a juicer and stored at $-18 \pm 1^{\circ} \mathrm{C}$.

\subsection{Yeast Strains}

Pure cultures of Saccharomyces cerevisiae Lalvin EC-1118 (EC1118) or Torulaspora delbrueckii UMY196 were used for the fermentation trials. S. cerevisiae EC1118 is a commercial yeast strain commonly employed for vinification (Lallemand Inc., Montreal, Quebec, Canada). T. delbrueckii UMY196 is part of the yeast culture collection of the University of Milan (Italy), and it was isolated from wine. Cells were maintained in yeast extract peptone dextrose (YPD) medium (10 g/L yeast extract, $20 \mathrm{~g} / \mathrm{L}$ peptone, $20 \mathrm{~g} / \mathrm{L}$ glucose, $\mathrm{pH}$ 5.5) supplemented with $20 \%(v / v)$ glycerol at $-80{ }^{\circ} \mathrm{C}$. Cell pre-cultures were obtained by inoculating $1 \%(v / v)$ glycerol stock freeze culture in YPD broth maintained at $30{ }^{\circ} \mathrm{C}$ for $24-48 \mathrm{~h}$ in aerobiosis. The final biomass was determined by optical density (OD) at $600 \mathrm{~nm}$. For the inocula, cells from the pre-culture were centrifuged at $3500 \mathrm{rpm}$ for $15 \mathrm{~min}$ (Hettich, ROTINA 380R, Tuttlingen, 
Germany), collected, and washed once with $0.9 \%(w / v) \mathrm{NaCl}$. The grape/fruit musts were inoculated at $0.1 \pm 0.05$ OD $600 \mathrm{~nm}$ corresponding to about $1 \times 10^{6} \mathrm{CFU} / \mathrm{mL}$.

\subsection{Fermentation Trials}

Grape/fruit-based drinks were produced through alcoholic fermentation (AF). Must/fruit juice mixes were tested in proportions of 80:20 $(v / v)$ and 60:40 $(v / v)$ for each must and juice collected, for a total of 48 grape/fruit-based drinks. The level of readily assimilable nitrogen (RAN) was measured in must/fruit juice mixes. If necessary, ammonium sulfate was added prior to the yeast inoculum adjusting the RAN content at $200 \mathrm{mg} / \mathrm{L}$. The AF was carried out in a flask $(200 \mathrm{~mL})$ at $25 \pm 1{ }^{\circ} \mathrm{C}$ and it was daily monitored by weight loss until no weight change was observed after two consecutive days. At the end of $\mathrm{AF}$, the drinks were centrifuged at $5000 \times \mathrm{g}$ for $20 \mathrm{~min}$ at $10{ }^{\circ} \mathrm{C}$ (Beckman, CA, USA), removing the yeast cells, followed by the addition of potassium metabisulfite $(50 \mathrm{mg} / \mathrm{L})$, before being stored at $4 \pm 1{ }^{\circ} \mathrm{C}$.

Based on the sensory evaluation, triplicate fermentation of the most pleasant grape/fruit-based drink was carried out in batches $(600 \mathrm{~mL})$ at $25 \pm 1{ }^{\circ} \mathrm{C}$ and in a glass demijohn $(4 \mathrm{~L})$ at $18 \pm 1{ }^{\circ} \mathrm{C}$, following the same procedure described for the flask trials.

\subsection{Microbial and Chemical Analysis}

The cell enumeration, content of sugars (glucose and fructose), total acidity, $\mathrm{pH}$, and organic acids were determined in both must/fruit juice mix and grape/fruit-based drinks, while ethanol was also evaluated in grape/fruit-based drinks. The ethanol yield (\%) was calculated as the molar ratio between the consumed sugars and ethanol produced.

Colony-forming unit (CFU/mL) enumeration was obtained for yeasts and lactic acid bacteria (LABs). The spreading dual plating $(100 \mu \mathrm{L})$ of useful decimal serial dilutions was carried out on Wallerstein Laboratory (WL) nutrient agar medium (Scharlau, Spain), after 2-4 days of incubation time at $30^{\circ} \mathrm{C}$ in aerobiosis for yeasts. LABs were enumerated on De Man, Rogosa and Sharpe (MRS; (BD Difco, Thermo-Fisher Scientific, Waltham, MA, USA) solid medium (1.5\% $w / v$ agar agar) at $6.2 \mathrm{pH}$, supplemented with $20 \%(v / v)$ apple juice and $0.01 \%(w / v)$ cycloheximide; cells were grown in anaerobic conditions at $30^{\circ} \mathrm{C}$ for $7-10$ days.

Ethanol, glucose, and fructose were determined using a Megazyme ethanol, D-fructose/D-glucose assay kit according to the manufacturer's instructions. The total acidity was determined by titration up to $\mathrm{pH} 7$ in accordance with the method OIV-MA-AS313-01 [29]. The RAN was quantified by the formol number with titration at $\mathrm{pH} 8.5$ [30]. The quantification of methanol was carried out by Enoconsulting (Erbusco, BS, Italy), an ISO 9000-accredited laboratory, through gas chromatography coupled with a flame ionization detector. The organic acids were quantified as described by Falqué López and Fernández Gómez [31] with some modifications. An Acquity HClass UPLC (Waters, Milford, MA, USA) system equipped with a photo diode array detector 2996 (Waters) was used. Chromatographic separations were performed with a Hypersil BDS C $8250 \times 4.6 \mathrm{~mm}, 5 \mu \mathrm{m}$ particle size (Alltech, Deerfield, IL, USA). The separation was carried out in isocratic conditions using sulfuric acid $(0.01 \mathrm{~N})$ at a flow rate of $0.8 \mathrm{~mL} / \mathrm{min}$, and the column temperature was $25^{\circ} \mathrm{C}$. Calibration curves were obtained for tartaric, malic, lactic, citric, acetic, and succinic acids at concentrations of $0.1-10 \mathrm{~g} / \mathrm{L}$. Quantification was performed according to the external standard method. Data acquisition and processing were carried out with Empower 2 software (Waters) at $210 \mathrm{~nm}$. The free and glycoconjugate aromas were determined as reported by Fracassetti, Gabrielli, Corona, and Tirelli [32]. Based on the known perception thresholds, the odor activity values (OAVs) were calculated as the ratio between the aroma concentration and its perception threshold.

\subsection{Sensory Analysis and Acceptability of Grape/Fruit-Based Drinks}

The sensory analysis was carried out for each grape/fruit-based drink obtained in flask trials and batch experiments. For the latter, the aroma profile was evaluated during the AF for a total of four 
samplings. Qualitative sensory analysis and the test of consumer acceptance were carried out for the experiment in a glass demijohn.

For flask and batch experiments, a panel of eight experienced judges (five females, three males) was enrolled. The scored descriptors included acidity, sweetness, bitterness, fruitiness, and the overall acceptability using a $10-\mathrm{cm}$ line scale.

For microvinification in a demijohn, a panel composed of 10 experienced judges (five females, five males) identified the attributes by the consensus method [33], which were scored by a nine-point scale with nine being the highest intensity. The quantitative profile was performed for the kiwi-based drink with and without added sugar. The discriminant capacity of the judges was set at $20 \%$ and the replicability was set at $75 \%$.

The test of consumer acceptability was carried out on the kiwi-based drink considering 100 consumers. A five-point scale was used with five being the highest intensity. The attributes were related to the visual, olfactory, taste, and global pleasantness, including the overall acceptability. Further questions were related to the eventual purchase of the beverage tasted and the indication of additional comments.

\subsection{Statistical Analysis}

One-way ANOVA was determined using SPSS Win 12.0 program (SPSS Inc., Chicago, IL). The equations of the calibration curves were assessed by linear regression analysis. Differences were evaluated by the $t$-test, and the significances were set at a value of $p<0.05$.

\section{Conclusions}

The present study proposes an innovative alcoholic beverage obtained by co-fermenting, using T. delbrueckii yeast, Cabernet Sauvignon must and kiwi juice in a proportion of 60:40. To the best of our knowledge, this is the first time such a product appears in literature. The feasibility of co-fermenting grape must and fruit juice was proven, leading to the production of a novel food resulting acceptable from the sensory point of view. The acceptability and approval expressed by potential consumers suggests that this drink could represent a valid strategy for the "re-use" of both kiwi and grape/must surplus, combining a sustainable approach with innovation in the alcoholic beverage field. The production of this alcoholic beverage can provide benefits to both wineries and farmers, since the losses of grape/wine and fruit can be limited and a longer use of the fermentation compartment of the cellar can be achieved. The proposed Cabernet Sauvignon/kiwi drink can be considered a pleasant beverage with low alcohol content, indicated as an aperitif. Future developments foresee a scale-up in the production of this drink, eventually using the equipment already present in a cellar to produce kiwi juice without compromising the quality of the juice, and managing the fermentation in order to preserve the desired residual sugar attenuating the acidity.

Supplementary Materials: The following are available online at http://www.mdpi.com/2218-1989/9/5/86/s1: Table S1: Ethanol yield in the flask fermentation trials fermented with Saccharomyces cerevisiae EC1118 (S. c.) and T. delbrueckii UMY196 (T. d.); Table S2: Chemical parameters determined for the fermentation trial with must/cherry juice fermented with S. cerevisiae EC1118 (S. c.) and T. delbrueckii UMY196 (T. d.); Table S3: Chemical parameters determined for the fermentation trial with must/peach juice fermented with S. cerevisiae EC1118 (S. c.) and T. delbrueckii UMY196 (T. d.); Table S4: Chemical parameters determined for the fermentation trial with must/strawberry juice fermented with Saccharomyces cerevisiae EC1118 (S. c.) and T. delbrueckii UMY196 (T. d.).

Author Contributions: Conceptualization, I.V.; methodology, I.V. and D.F.; software, D.F.; validation, P.B. and O.C.; formal analysis, D.F. and O.C.; investigation, I.V., D.F., and P.B.; resources, P.B.; data curation, D.F. and R.F.; writing — original draft preparation, I.V.; writing — review and editing, I.V., D.F., and R.F.; supervision, I.V. and D.F.; project administration, I.V.; funding acquisition, I.V.

Funding: Ileana Vigentini and Daniela Fracassetti are grateful to Piano di Sostegno alla Ricerca 2015/2017-Linea 2-Università degli Studi di Milano.

Acknowledgments: We are grateful to the Mirabella and Cantina Sociale Bergamasca wineries for providing the Chardonnay and Cabernet Sauvignon musts, respectively. 
Conflicts of Interest: The authors declare no conflicts of interest.

\section{Appendix A}

Table A1. Summary of the chemical parameters (residual sugars, ethanol, $\mathrm{pH}$, total acidity, organic acid profile) for the must/fruit-based drink. The data related to organic acids are reported in Figure A1.

\begin{tabular}{|c|c|}
\hline Fruit-Based Drink & Chemical Characteristic of the Final Product \\
\hline $\begin{array}{l}\text { Cherry-based drinks } \\
\text { (Table S2) }\end{array}$ & $\begin{array}{l}\text { Residual sugars: }<1.0 \pm 0.0 \mathrm{~g} / \mathrm{L} \text { in all the combinations. } \\
\text { Ethanol: It was the highest compared to the others investigated combinations, reaching the maximum in } \\
\text { combination with Cabernet Sauvignon } 80: 20(12.7 \pm 0.1 \%(v / v) \text { for } S . \text { cerevisiae and } 12.3 \pm 0.7 \%(v / v) \text { for } T \text {. delbrueckii). } \\
\text { pH: } 3.27 \pm 0.02 \text { to } 3.54 \pm 0.00 \text {. } \\
\text { Total acidity: Higher in beverages produced with T. delbrueckii. } \\
\text { Organic acids: Malic acid decreased during the alcoholic fermentation, although it was the major organic acid in the } \\
\text { drinks produced. Acetic acid was found only in the drinks fermented with T. delbrueckii (maximum } 1.8 \pm 0.0 \mathrm{~g} / \mathrm{L} \text { in } \\
\text { the } 80: 20 \text { mix with Cabernet Sauvignon). }\end{array}$ \\
\hline $\begin{array}{l}\text { Kiwi-based drinks } \\
\text { (Table 1) }\end{array}$ & $\begin{array}{l}\text { Residual sugars: Higher in the mixes Cabernet Sauvignon fermented by T. delbrueckii ( } 71 \pm 2 \mathrm{~g} / \mathrm{L} \text { and } 52 \pm 17 \mathrm{~g} / \mathrm{L} \text { for } \\
\qquad 80: 20 \text { and } 60: 40 \text { combinations, respectively). } \\
\text { Ethanol: } 6.5 \pm 0.3 \%(v / v) \text { and } 7.0 \pm 1.3 \%(v / v) \text { for mixes } 80: 20 \text { and } 60: 40 \text {, respectively, fermented by T. delbrueckii in } \\
\text { comparison to the respective fermented drinks with S. cerevisiae }(10.8 \pm 0.5 \%(v / v) \text { for } 80: 20 \text { and } 9.6 \pm 0.3 \%(v / v) \text { for } \\
\quad 60: 40 \text {. } \\
\text { pH: Slightly higher in drinks fermented with T. delbrueckii }(3.13 \pm 0.08 \text { to } 3.50 \pm 0.01) \text { in the mix } 60: 40 \text { with } \\
\text { Chardonnay must. } \\
\text { Total acidity: } 11.5 \pm 0.7 \text { to } 16.3 \pm 0.3 \mathrm{~g} \text { tartaric acid/L in the tests with T. delbrueckii. } \\
\text { Organic acids: The most abundant was citric acid, deriving from kiwi juice. Succinic acid was detected at the end of } \\
\text { fermentation, while acetic acid was found only in drinks based on Chardonnay must and fermented by T. delbrueckii. }\end{array}$ \\
\hline $\begin{array}{l}\text { Peach-based drinks } \\
\quad \text { (Table S3) }\end{array}$ & $\begin{array}{c}\text { Residual sugars: }<1.0 \pm 0.0 \mathrm{~g} / \mathrm{L} \text { in all the combinations. } \\
\text { Ethanol: Higher in the } 80: 20 \text { combinations for both musts and yeasts used. } \\
\text { pH: Slightly higher in drinks fermented with T. delbrueckii ( } 3.30 \pm 0.01 \text { to } 3.72 \pm 0.01 \text { ) in the mix } 60: 40 \text { with } \\
\text { Chardonnay must. } \\
\text { Total acidity: Slight differences between drinks fermented with } S \text {. cerevisiae and T. delbrueckii. } \\
\text { Organic acids: Both tartaric and malic acids decreased during the fermentation. The drinks contained succinic acid } \\
\text { (highest amount } 1.40 \pm 0.23 \mathrm{~g} / \mathrm{L} \text { ), as well as acetic acid (highest amount } 0.98 \pm 0.03 \mathrm{~g} / \mathrm{L} \text { ). }\end{array}$ \\
\hline $\begin{array}{l}\text { Strawberry-based drinks } \\
\text { (Table S4) }\end{array}$ & $\begin{array}{l}\text { Residual sugars: }<2.5 \pm 1.5 \mathrm{~g} / \mathrm{L} \text {, except for the trial with Cabernet Sauvignon must at the proportion } 80: 20 \text { fermented } \\
\text { by T. delbrueckii in which the residual sugar was } 44 \pm 5 \mathrm{~g} / \mathrm{L} \text {. } \\
\text { Ethanol: From } 4.4 \pm 1.0 \%(v / v) \text { in the trial with Chardonnay must at the proportion } 60: 40 \text { fermented by T. delbrueckii } \\
\text { to } 9.9 \pm 0.9 \%(v / v) \text { in the test with Cabernet Sauvignon must at the proportion } 80: 20 \text { fermented by S. cerevisiae. The } \\
\text { higher concentrations were detected in drinks obtained from mixes with Cabernet Sauvignon, attributable to the } \\
\text { higher sugar content. } \\
\text { pH: No significant differences among the different combinations must/strawberry juice (range } 2.97 \pm 0.00 \text { to } \\
\quad 3.22 \pm 0.02) \text {. } \\
\text { Total acidity: Higher in the fermented mixes with T. delbrueckii. } \\
\text { Organic acids: A significant decrease in tartaric acid occurred. Acetic acid was in the range } 1.3 \pm 0.0 \mathrm{~g} / \mathrm{L} \text { to } \\
2.4 \pm 0.3 \mathrm{~g} / \mathrm{L} \text {, except for the drinks based on Chardonnay must and fermented with T. delbrueckii. }\end{array}$ \\
\hline
\end{tabular}
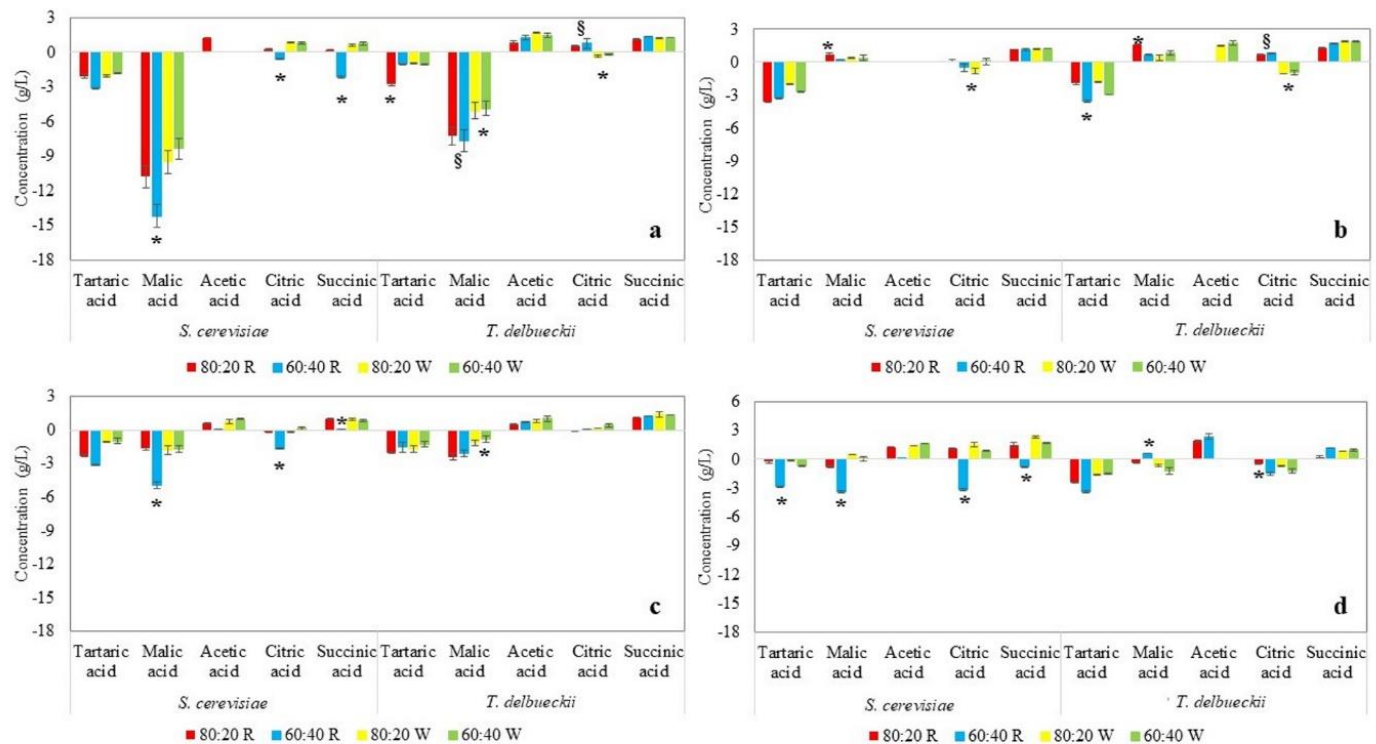

Figure A1. Evolution of organic acids (g/L) at the end of fermentations for the drinks produced in the flask experiment for (a) cherry, (b) kiwi, (c) peach, and (d) strawberry juices. Data were calculated as the difference between sampling at the end of alcoholic fermentation and at the initial time. Error bars indicate the standard deviation among the replicates. 


\section{References}

1. McGovern, P.E.; Jalabadze, M.; Batiuk, S.; Callahan, M.P.; Smith, K.E.; Hall, G.R.; Kvavadze, E.; Maghradze, D.; Rusishvili, N.; Bouby, L.; et al. Early Neolithic wine of Georgia in the South Caucasus. Proc. Natl. Acad. Sci. USA 2017, 114, E10309-E10318. [CrossRef]

2. McGovern, P.E.; Zhang, J.; Tang, J.; Zhang, Z.; Hall, G.R.; Moreau, R.A.; Nunez, A.; Butrym, E.D.; Richards, M.P.; Wang, C.; et al. Fermented beverages of pre- and proto-historic China. Proc. Natl. Acad. Sci. USA 2004, 101, 17593-17598. [CrossRef] [PubMed]

3. Jagtap, U.B.; Bapat, V.A. Wines from fruits other than grapes: Current status and future prospectus. Food Biosci. 2015, 9, 80-96. [CrossRef]

4. State of the Viticulture World Market. April 2018. Available online: http://www.oiv.int/public/medias/5958/ oiv-state-of-the-vitiviniculture-world-market-april-2018.pdf (accessed on 19 March 2019).

5. Swami, S.B.; Thakor, N.J.; Divate, A.D. Fruit Wine Production: A Review. J. Food Res. Technol. 2014, 2, 93-100.

6. Kosseva, M.R.; Joshi, V.K.; Panesar, P.S. Science and Technology of Fruit Wine Production, 2nd ed.; Kosseva, M.R., Joshi, V.K., Panesar, P.S., Eds.; Elsevier: London, UK, 2016.

7. Duarte, F.W.; Dias, D.R.; Oliveira, J.M.; Teixeira, J.A.; de Almeida e Silva, J.B.; Schwan, R.F. Characterization of different fruit wines made from cacao, cupuassu, gabiroba, jaboticaba and umbu. LWT Food Sci. Technol. 2010, 43, 1564-1572. [CrossRef]

8. Coelho, E.; Vilanova, M.; Genisheva, A.; Oliveira, J.M.; Teixeira, J.A.; Domingues, L. Systematic approach for the development of fruit wines from industrially processed fruit concentrates, including optimization of fermentation parameters, chemical characterization and sensory evaluation. LWT Food Sci. Technol. 2015, 62, 1043-1052. [CrossRef]

9. Lipinski, B.; Hanson, C.; Lomax, J. Reducing food loss and waste. In Installment of "Creating a Sustainable Food Future"; Working Paper; World Resources Institute: Washington, DC, USA, 2013. Available online: http://www.worldresourcesreport.org (accessed on 20 March 2019).

10. Belda, I.; Navascués, E.; Marquina, D.; Santos, A.; Calderon, F.; Benito, S. Dynamic analysis of physiological properties of Torulaspora delbrueckii in wine fermentations and its incidence on wine quality. Appl. Microbiol. Biotechnol. 2015, 99, 1911-1922. [CrossRef]

11. Benito, S. The impact of Torulaspora delbrueckii yeast in winemaking. Appl. Microbiol. Biotechnol. 2018, 102, 3081-3094. [CrossRef]

12. Redzepovic, S.; Orlic, S.; Majdak, A.; Kozina, B.; Volschenk, H.; Viljoen-Bloom, M. Differential malic acid degradation by selected strains of Saccharomyces during alcoholic fermentation. Int. J. Food Microbiol. 2003, 83, 49-61. [CrossRef]

13. Chidi, B.S.; Bauer, F.F.; Rossouw, D. Organic acid metabolism and the impact of fermentation practices on wine acidity: A review. S. Afr. J. Enol. Vitic. 2018, 39, 1-15. [CrossRef]

14. McMahon, K.M.; Diako, C.; Aplin, J.; Mattinson, D.S.; Culver, C.; Ross, C.F. Trained and consumer panel evaluation of sparkling wines sweetened to brut or demi sec residual sugar levels with three different sugars. Food Res. Int. 2017, 99, 173-185. [CrossRef]

15. Ribéreau-Gayon, J.; Glories, Y.; Maujean, A.; Dubourdieu, D. Handbook of Enology. The Microbiology of Wine and Vinifications, Volume 2, 3rd ed.; Wiley: New York, NY, USA, 2006.

16. Sumby, K.M.; Grbin, P.R.; Jiranek, V. Microbial modulation of aromatic esters in wine: Current knowledge and future prospects. Food Chem. 2010, 121,1-16. [CrossRef]

17. Vigentini, I.; Maghradze, D.; Petrozziello, M.; Bonello, F.; Mezzapelle, V.; Valdetara, F.; Failla, O.; Foschino, R. Indigenous Georgian wine-associated yeasts and grape cultivars to edit the wine quality in a precision oenology perspective. Front. Microbiol. 2016, 7, 352. [CrossRef] [PubMed]

18. López, R.; Ferreira, V.; Hernández, P.; Cacho, J.F. Identification of impact odorants of young red wines made with Merlot, Cabernet Sauvignon and Grenache grape varieties: A comparative study. J. Sci. Food Agric. 1999, 79, 1461-1467. [CrossRef]

19. Jordán, M.J.; Margaría, C.A.; Shaw, P.E.; Goodner, K.L. Aroma active components in aqueous kiwi fruit essence and kiwi fruit puree by GC-MS and multidimensional GC/GC-O. J. Agric. Food Chem. 2002, 50, 5386-5390. [CrossRef] [PubMed]

20. Dragone, G.; Mussatto, S.I.; Oliveira, J.M.; Teixeira, J.A. Characterisation of volatile compounds in an alcoholic beverage produced by whey fermentation. Food Chem. 2009, 112, 929-935. [CrossRef] 
21. Escudero, A.; Campo, E.; Farina, L.; Cacho, J.; Ferreira, V. Analytical characterization of the aroma of five premium red wines. Insights into the role of odor families and the concept of fruitiness of wines. J. Agric. Food Chem. 2007, 55, 4501-4510. [CrossRef] [PubMed]

22. Francis, I.L.; Newton, J.L. Determining wine aroma from compositional data. Aust. J. Grape Wine Res. 2005, 11, 114-126. [CrossRef]

23. Gamero, A.; Ferreira, V.; Pretorius, I.S.; Querol, A. Wine, beer and cider: Unrevelling the aroma profile. In Molecular Mechanisms in Yeast Carbon Metabolism; Piskur, J., Compagno, C., Eds.; Springer: Berlin/Hidelberg, Germany, 2014; pp. 261-297.

24. Gómez-Míguez, M.J.; Cacho, J.F.; Ferreira, V.; Vicario, I.M.; Heredia, F.J. Volatile components of Zalema white wines. Food Chem. 2007, 100, 1464-1473. [CrossRef]

25. Moreno, J.A.; Zea, L.; Moyano, L.; Medina, M. Aroma compounds as markers of the changes in sherry wines subjected to biological ageing. Food Control 2005, 16, 333-338. [CrossRef]

26. Peinado, R.A.; Mauricio, J.C.; Moreno, J. Aromatic series in sherry wines with gluconic acid subjected to different biological aging conditions by Saccharomyces cerevisiae var. capensis. Food Chem. 2006, 94, 232-239. [CrossRef]

27. Hernández-Orte, P.; Cersosimo, M.; Loscos, N.; Cacho, J.; Garcia-Moruno, E.; Ferreira, V. The development of varietal aroma from non-floral grapes by yeasts of different genera. Food Chem. 2008, 107, 1064-1077. [CrossRef]

28. European Community. Regulation (EC) No 110/2008 of the European Parliament and of the council on the definition, presentation, labelling and the protection of geographical indications of spirit drinks and repealing Council Regulation (EEC) No. 1576/89. Off. J. Eur. Union 2008, L 39/16, 16-54.

29. Resolution Oeno 551-2015. Compendium of International Methods of Analysis-OIV. Method OIV-MA-AS313-01 for the Determination of Total Acidity. Available online: http://www.oiv.int/en/technical-standards-anddocuments/methods-of-analysis/compendium-of-international-methods-of-analysis-of-wines-and-musts-2vol (accessed on 29 April 2019).

30. Cavaglioni, A.; Ferrari, S. Confronto tra alcuni metodi per la determinazione dell'azoto prontamente assimilabile. Vignevini 2002, 11, 119-123.

31. Falqué López, E.; Fernández Gómez, E. Simultaneous determination of the major organic acids, sugars, glycerol, and ethanol by HPLC in grape musts and white wines. J. Chromatogr. Sci. 1996, 34, 254-257. [CrossRef]

32. Fracassetti, D.; Gabrielli, M.; Corona, O.; Tirelli, A. Characterisation of Vernaccia Nera (Vitis vinifera L.) grapes and wine. S. Afr. J. Enol. Vitic. 2017, 38, 72-81.

33. ISO 11035:1994. Sensory Analysis-Identification and Selection of Descriptors for Establishing a Sensory Profile by a Multidimensional Approach; ISO: Geneva, Switzerland, 1994. 